\title{
Barreras para la integración de buenas prácticas con TIC. Estudio de caso
}

\section{Barrers for ICT best practices. Case study}

\author{
Siria Padilla Partida ${ }^{1}$, Carlos Iván Moreno ${ }^{2}$, Rosario Hernández Castañeda ${ }^{3}$ \\ ${ }^{1}$ Departamento de Políticas Públicas, Universidad de Guadalajara, México (siriapadilla@gmail.com) \\ 2Departamento de Políticas Públicas, Universidad de Guadalajara, México (carlosivan.moreno@gmail.com) \\ ${ }^{3}$ Departamento de Ciencias Sociales y Jurídicas, Universidad de Guadalajara, México (rosariohd2000@ gmail.com)
}

\section{RESUMEN:}

Esta investigación analiza las barreras u obstáculos para la integración de las buenas prácticas educativas con TIC, en la segunda más grande universidad pública de México: La Universidad de Guadalajara. Se analiza el caso del Centro Universitario de Ciencias Económico Administrativas (CUCEA), el cual es uno de los 15 centros universitarios que integran la red universitaria de la UdeG. Para esta investigación fue diseñado un cuestionario cerrado, tipo rubrica, con una escala de 5 puntos que iba desde no se ha iniciado hasta consolidado, para poder identificar cuáles eran los principales obstáculos percibidos por los profesores para integrar las TIC en las prácticas educativas. El cuestionario estuvo dividido en dos partes, la primera exploraba las barreras atribuidas a la institución educativa, y la segunda, las barreras atribuidas a los profesores. El muestreo fue representativo y aleatorio, con una muestra de 210 profesores de un total de 460. Los resultados identificaron como los mayores obstáculos: La falta de incentivos para integrar las TIC en la enseñanza con 382 puntos de un total de 1055, la falta de flexibilidad curricular con 483 puntos, y por último, la falta de confianza en la educación a distancia con 583 puntos.

PALABRAS CLAVE: BARRERAS, TIC, EDUCACIÓN SUPERIOR, BUENAS

\section{ABSTRACT:}

This research analyzes the barriers or obstacles to the integration of ICT's best educational practices in the second largest public university in Mexico: The University of Guadalajara. A case study was conducted at the University Center for Economic and Administrative Sciences (CUCEA). CUCEA is one of 15 centers that integrate the academic network of the University of Guadalajara. For this research, a closed questionnaire, type rubric, was designed with a five-point scale ranging from "not started" to "consolidated," in order to identify the main obstacles, as perceived by the faculty, to the integration of ICT into existing educational practices. The questionnaire was divided into two parts: The first part explored the barriers attributed to the educational institution, and the second part explored the barriers attributed to teachers. The sample was representative and random, with a sample of 210 teachers out of a total of 460 . The results identified as major obstacles: the lack of incentives to integrate ICT into teaching ( 382 points out of 1055), the lack of flexibility of the curriculum (483), and, finally, the lack of trust in distance education (583).

KEYWORDS: BARRIERS, ICT, HIGHER EDUCATION. 


\section{INTRODUCCIÓN}

La integración de las TIC a la educación superior no es sólo un problema de transferencia y dotación de tecnologías a los centros educativos, no se trata únicamente de que profesores y estudiantes tengan a su alcance computadoras, redes, y software, sin duda, se trata de un primer paso para empezar a crear las condiciones adecuadas para un uso regular y cotidiano de las TIC pero nunca suficiente por sí mismo.

En la integración de las TIC intervienen muchas aristas que complejizan y dificultan este proceso: problemas en la gestión y organización del centro educativo, concepciones tradicionales de cómo se entiende y realiza el proceso de enseñanza y aprendizaje en las aulas, así como las actitudes del profesorado hacia las tecnologías; además, entre estos tres elementos existen múltiples interacciones que problematizan aún más esta red.

Este artículo aborda los obstáculos que enfrentan las Instituciones de Educación Superior (IES) para hacer "visibles" las computadoras en las prácticas educativas. El estudio de las barreras es, por otra parte, la realización de un diagnóstico que nos permite reconocer e identificar cuáles son los problemas que enfrentan las IES en la implementación de las políticas educativas relacionadas con la integración de las TIC a las aulas. Por supuesto, ese diagnóstico proporciona elementos fundamentales para iniciar procesos de acción, toma de decisiones que ayuden a orientar las políticas y los programas institucionales que hacen posible la transformación de las universidades, de ahí su importancia.

\subsection{Tendencias actuales}

El Plan Nacional de Desarrollo (2007-2012) en materia de educación y tecnologías, partía del reconocimiento de la importancia que tiene la sociedad del conocimiento en el desarrollo de las naciones. Las tecnologías se ven como una de las estrategias para lograr que los estudiantes puedan acceder a esa sociedad del conocimiento a partir del acceso, aplicación e incorporación de las tecnologías a los procesos educativos, así en el objetivo 11 se busca "Impulsar el desarrollo y utilización de las nuevas tecnologías en el sistema educativo para apoyar la inserción de los estudiantes en la sociedad del conocimiento y ampliar sus capacidades para la vida" (PND, p. 187).

En correspondencia el Plan Sectorial de Educación (PSE, 2007-2012) contemplaba en su objetivo 3 "impulsar el desarrollo y utilización de las tecnologías de la información y comunicación en el sistema educativo para apoyar el aprendizaje de los estudiantes, ampliar sus competencias para la vida y favorecer su inserción en la sociedad del conocimiento" (p. 11).

En lo que respecta a educación superior, las TIC sirven para "mejorar los ambientes y proceso de aprendizaje, la operación de redes de conocimiento y el desarrollo de proyectos intra e interinstitucionales" (p.40), en otras palabras la ES articula y pone en marcha las acciones que hacen posible la sociedad del conocimiento.

Por su parte, el Plan Nacional de Desarrollo actual (PND, 2013-2018) es crítico con respecto al uso e incorporación de las TIC a la educación. Las TIC, dice en su diagnóstico, no han comprobado ser un factor para el desarrollo de la calidad educativa, no existe evidencia empírica suficiente que demuestre una correlación positiva entre el uso de las TIC y la mejora de los procesos de enseñanza aprendizaje, sin embargo el plan reconoce que se debe promover la incorporación de las tecnologías en los procesos de enseñanza aprendizaje $\mathrm{y}$, se establece la necesidad de crear una estrategia nacional de formación en y con TIC.

Por otro lado, el Plan Sectorial de Educación (2013-2018) en su objetivo 2 busca fortalecer la calidad y pertinencia de la educación media superior y superior $\mathrm{y}$, la formación para el trabajo, a fin de que contribuyan al desarrollo de México; así el objetivo 2.6. busca "aprovechar las tecnologías de la información y comunicación para el fortalecimiento de la educación media superior y superior" como es el apoyar los programas en las modalidades de educación continua, abierta y a distancia, o bien, apoyar el uso de las TIC en la modalidad presencial con la incorporación de unidades de aprendizaje en línea. En este contexto se plantea entonces la necesidad de equipar y dotar de plataformas tecnológicas pertinentes a los centros educativos y capacitar a los docentes.

Los lineamientos anteriores son el marco de fondo a partir del cual las IES desarrollan sus propios planes y programas en materia de TIC. En el caso del CUCEA, el Plan de Desarrollo Institucional (PDI, 2002-2010) plantea que las tecnologías pueden potencializar la transformación de las prácticas educativas a partir del uso de las TIC.

Alentado por las nuevas posibilidades tecnológicas, se hace necesario un proceso que inaugure un modelo educativo acorde con la nueva era. La innovación de la docencia debe presentarse tanto a nivel de licenciatura 
como de posgrado y consiste en el desarrollo de nuevos modelos de interacción y de aprendizaje que aprovechen las nuevas tecnologías de la información y la gestión del conocimiento. La estructuración de ambientes de aprendizaje representa un reto importante ya que el uso de herramientas por sí mismo no es suficiente, sino que debe ir acompañado de un replanteamiento innovador de los procesos académicos para superar el paradigma tradicional (p.8).

El CUCEA busca, a través de las TIC, introducir nuevos modelos de enseñanza que ayuden a transformar las prácticas educativas tradicionales que se realizan en la institución. Se reconoce que el uso de las herramientas, por sí mismo, no es capaz de modificar las prácticas educativas, éstas deben acompañarse de un modelo educativo que les otorgue sentido y forma. De igual manera, se señala la posibilidad de desarrollar programas no convencionales: mixtos y a distancia con apoyo de las TIC.

Este trabajo analiza cuáles son los obstáculos en materia de TIC que dificultan la implementación de las políticas educativas. Se entiende, que la evolución acelerada de las tecnologías, con su cambio frenético, tienen repercusiones en el ritmo de integración de las TIC, los nuevos modelos desplazan los antiguos y, las nuevas versiones hacen obsoletas las anteriores, pero existen otras dinámicas que tienden a inmovilizar las instituciones y aletargar los cambios planteados ¿cuáles son esos obstáculos? es la pregunta que se plantea este trabajo de investigación.

De acuerdo con Bingimlas (2009) la ausencia de las TIC en los centros educativos se debe a la existencia de barreras extrínsecas (atribuibles al centro educativo) $\mathrm{y}$, las barreras intrínsecas, (atribuidas a los profesores). Uno de los principales obstáculos que se reportan a nivel internacional es el atraso en la infraestructura como tener un número insuficiente de computadoras por estudiantes, la ausencia de software especializado, la falta de actualización de programas, la existencia de computadoras en mal estado y la falta de un equipo de apoyo especializado (Barrantes, Casas y Luengo, 2011; Cabero, 2001; Pelgrum, 2001).

Otra barrera destacada es la escasa formación de los profesores en el uso de las TIC y, su aplicación didáctica, ésta última orientación es básica para visualizarlas como herramientas estratégicas para el desarrollo de competencias y el desarrollo de habilidades (Area, 2002; Bingimlas, 2009; Cabero, 2001; Pelgrum, 2001).

Una tercera barrera, reportada por la literatura es la falta de tiempo, que incluye la carencia de 1 mismo para planificar el trabajo educativo, para el desarrollo de materiales educativos, para la identificación de recursos en internet $\mathrm{y}$, para la identificación sobre la forma en cómo estos recursos y materiales pueden ser evaluados (Barrantes, Casas y Luengo, 2011; Jones, 2004; Pelgrum, 2001).

Con relación a las barreras culturales, Area (2002) menciona las prácticas pedagógicas tradicionales, las cuales se instituyen como prácticas vigentes, difíciles de modificar y superar y, que se ven soportadas por la organización del curriculo, (disciplinar y centrado en contenidos), los programas $\mathrm{y}$, la integración disciplinar de las academias. A ello se agrega la tendencia en América Latina a minusvalorar la educación a distancia; para muchos profesores las tecnologías no aportan nada que no se pueda hacer de manera presencial, las tecnologías hacen que se pierda la riqueza y calidad de las interacciones cara a cara (Valzacchi, 2010; Vianney y Lupión (2010). Sin embargo estas barreras culturales no es correcto atribuirlas al centro educativo, se trata de imaginarios compartidos de entender la acción docente.

Con respecto a las barreras intrínsecas se reportan las actitudes del profesorado ante las TIC. Las actitudes negativas representan serios obstáculos que determinan, incluso, su exclusión del aula y, están asociadas a una auto-percepción negativa sobre el dominio de las TIC. Si se tiene una percepción de una autoeficacia negativa los profesores serán renuentes a utilizar las herramientas pues su utilización estará asociada a sentimientos de frustración, ansiedad y angustia (Barrantes, Casas y Luengo, 2011; Ramírez, Cañedo y Clemente, 2011).

En una investigación realizada por Olivarria y Carpio (2006) en la que participaron profesores de diversos programas a distancia a nivel latinoamericano sobre las desventajas que los participantes ven a los cursos a distancia (se trataba del programa Red Global del Aprendizaje para el Desarrollo GDLN) se identifican las siguientes:

1. La falta de experiencia en educación a distancia ha hecho que las universidades tengan que invertir una mayor cantidad de recursos adicionales a los previstos.

2. La segunda dificultad ha sido la imagen menor y de poca valía que acompaña a la educación a distancia, eso ha llevado a varias universidades a 
tener dudas sobre su involucramiento en este tipo de actividades.

3. No ha sido fácil involucrar a académicos en la educación a distancia, acostumbrados como lo han hecho durante toda su vida, a trabajar presencialmente. Adicionalmente, muchos de ellos no manejan el uso de las TIC.

Destacamos aquí, como relevante para este trabajo la falta de confianza en las modalidades no presenciales como un factor que puede tener un peso relevante en el desarrollo e integración de prácticas con TIC en la Universidad de Guadalajara.

\section{PLANTEAMIENTO DEL PROBLEMA}

De acuerdo con la revisión de la literatura y los antecedentes, los autores de este artículo buscan analizar cuáles son las principales barreras que identifican los docentes del Centro Universitario de Ciencias Económico Administrativas (CUCEA) para integrar las TIC a las prácticas educativas. El CUCEA cuenta con una infraestructura óptima que permite utilizar la PC, cañón, terminal de DVD y USB en cada aula. Además tiene jardines cibernéticos e internet en todo el campus que les permite conectarse todo el tiempo. Con respecto al staff se trabaja con una Coordinación de Tecnologías para el Aprendizaje y la Unidad de Diseño Educativo (UDE), ésta última responsable de apoyar a los profesores en el diseño de cursos y materiales en línea.

Dada la existencia de estos elementos tecnológicos y humanos de soporte, llama la atención que el CUCEA tenga tan poca respuesta en la integración de las plataformas tecnológicas a las prácticas educativas, así para el año 2013 se registraron sólo 12 cursos en línea en todo el centro universitario, tomando en cuenta que, para ese mismo año se contaba con 17,332 estudiantes, 16,270 en nivel superior y 1,062 en posgrado (CUCEA, 2014a).

Para esta investigación debe distinguirse entre curso y espacio en línea, de estos últimos el CUCEA reporta la existencia entre 200 a 300 materiales en el último año. Sin embargo, no se ha documentado hasta ahora ningún lineamiento para identificar, diferenciar y evaluar los espacios y cursos en línea. De facto, se identifica el curso en línea con la modalidad a distancia y, el espacio en línea con la modalidad presencial. En ambos casos se utiliza la plataforma moodle como apoyo. Básicamente lo que distinguiría uno de otro es que el curso en línea incorpora un diseño instruccional pedagógico y tecnológico para el logro de ciertos objetivos didácticos a desarrollarse en el aula virtual; por su parte el espacio en línea utiliza ciertas herramientas o materiales como apoyo al trabajo que se desarrolla en las sesiones presenciales. Esta falta de clarificación no es propia únicamente del CUCEA, existen diferencias de términos dentro de la propia universidad, así el Centro Universitario del Sur (CUSUR) por ejemplo, ha distinguido entre repositorio, espacio de apoyo y curso en línea, también ha establecido criterios específicos para evaluar cada uno de ellos (CUSUR, 2014). Por su parte el Centro Universitario de los Valles (2015) reconoce únicamente el material instruccional en línea, pero evalúa y clasifica dichos materiales en cuatro niveles de acuerdo con las herramientas que los profesores utilicen como apoyo a los procesos de enseñanza aprendizaje.

Cabe mencionar además que el Plan de Desarrollo 2002-2010 (CUCEA, 2002) del centro universitario contemplaba dos líneas de acción: la formación y capacitación de los docentes en TIC con el fin de innovar las prácticas educativas y, el diseño de programas no convencionales a partir del uso de las tecnologías. Por su parte, el nuevo Plan de Desarrollo del CUCEA, Visión 2030 (CUCEA, 2014a), contempla el uso intensivo de las TIC para el aprendizaje, el cual busca "asegurar un modelo educativo innovador, dinámico y flexible centrado en el proceso de enseñanza y aprendizaje y apoyado en las mejores prácticas pedagógicas, utilizando las nuevas tecnologías para el aprendizaje" (p. 3).

Tomando en consideración ambos planes, la institución ha promovido estrategias para incentivar el uso de las plataformas tecnológicas como apoyo a las prácticas educativas y, el diseño de cursos en línea. Igualmente, ha proporcionado capacitación docente en el uso de la plataforma moodle a los docentes del centro universitario. Sin embargo, a pesar de que existen buenas condiciones de infraestructura y de servicios de internet, así como apoyo en los servicios técnicos de apoyo a la docencia, existen pocos cursos en línea y, baja integración de las TIC a las prácticas educativas.

Por otra parte, tomando en cuenta la revisión de la literatura, se reconoce que las actitudes del profesorado hacia las TIC son positivas, es decir, una mayoría de los profesores tiene percepciones positivas sobre los beneficios que aportan las TIC a las prácticas educativas, sin embargo, esto no se ha 
traducido en un aumento de la utilización de los cursos en línea en las prácticas docentes.

Si esto es así, si los profesores de CUCEA tienen buenas actitudes hacia las TIC y, buena infraestructura, cabe preguntarse:

a. ¿Cuáles son las principales barreras que identifican los profesores en la integración de las TIC a las prácticas educativas?

b. ¿Qué peso tienen las barreras extrínsecas o atribuibles al centro en la ausencia de una integración de las TIC a las prácticas educativas?

c. ¿Qué peso tienen las barreras intrínsecas o atribuibles al profesor en la ausencia de una integración de las TIC a las prácticas educativas?

\section{DISEÑO METODOLÓGICO}

Para este trabajo de investigación se realiza una investigación estudio de caso, reconociendo que según Yin (2009) el estudio de caso es la estrategia adecuada cuando se requiere conocer a profundidad una organización, objeto o proceso específico con riqueza de detalle y narrativa. La unidad de análisis de este objeto de estudio son las barreras extrínsecas e intrínsecas.

Se utiliza como medio de acercamiento a las preguntas de investigación el diseño de un cuestionario cerrado, tipo rúbrica, el cual fue validado a través de la prueba Alfa de Cronbach y obtuvo 8.4 de confiabilidad. El cuestionario está dividido en barreras extrínsecas e intrínsecas, de acuerdo a la revisión de la literatura. Se aplicó una muestra aleatoria, de la cual se obtuvieron 211 profesores sobre una población de 460.

La aplicación del cuestionario se realizó a través de formatos electrónicos como google drive $\mathrm{y}$ survey monkey, sin embargo, fue necesario la aplicación de encuestas de manera presencial pues no fue posible conseguir la participación esperada. Del total de la muestra estimada se obtuvieron 211 cuestionarios, por lo cual se cumplió la muestra.

\subsection{Descripción del estudio de caso}

El Centro Universitario de Ciencias Económico Administrativas (CUCEA) forma parte de la Universidad de Guadalajara, el cual paso, en 1995, de ser Facultad de Ciencias EconómicoAdministrativas a convertirse en el centro temático de esa misma área. Para 2013 el centro contaba con 17,332 estudiantes, 16,270 en nivel superior y 1,062 en posgrado (CUCEAa, 2014). Actualmente se cuenta con 17, 683 estudiantes, 16,720 en nivel superior y 963 de posgrado (CUCEA, 2015). Tiene
27 programas educativos, 11 de licenciatura y 16 de posgrado.

Con respecto al staff y siguiendo las tendencias actuales sobre la integración de las TIC, en el año de 1999 se creó una Coordinación de Tecnologías para el Aprendizaje y, al mismo tiempo una serie de unidades de apoyo entre ellas, la Unidad de Multimedia Instruccional (2001), la Unidad de Cómputo y Telecomunicaciones para el Aprendizaje (2000), Unidad de Cursos en Línea y Educación a Distancia (2003) y, más recientemente (2011) la Unidad de Diseño Educativo (UDE).

A la UDE, por su parte, se le atribuyen las funciones siguientes (CUCEA, 2013):

- Asesorar el diseño instruccional de los cursos en línea.

- Desarrollar materiales educativos

- Gestionar información confiable y actual para los cursos en línea.

- Vigilar los derechos de autor de los recursos ofrecidos en los cursos en línea.

- Subir a la plataforma el curso en línea y generar espacios correspondientes para los profesores que lo impartirán.

- Generar espacios y cuentas de acceso a moodle para la comunidad universitaria.

- Capacitar a los docentes en todo lo referente al diseño y uso de cursos en línea y en la aplicación de las TIC para el aprendizaje.

Con la creación de la UDE en el año 2011 se reconoce que el apoyo anterior que se otorgaba a los profesores tenía un carácter técnico más que pedagógico, se daba capacitación y apoyo tecnológico a los profesores, pero se dejaba de lado la importancia del diseño instruccional y de la orientación pedagógica al docente para organizar el curso, seleccionar materiales, elegir formas de evaluación y estrategias docentes con TIC. La suplencia de estas carencias, sin embargo, no ha hecho posible aumentar el número de cursos en línea que se utilizan en el campus.

A partir del año 2012 calendario "B", se pone en marcha el proyecto de enseñanza de la asignatura de Tecnologías de la Información a todos los estudiantes del centro universitario, en este proyecto participan 38 profesores del departamento de Sistemas de Información bajo la modalidad "presencial enriquecida." El curso es obligatorio, tiene como objeto dotar de herramientas en TIC a los estudiantes en programas como Word, excell y páginas web. Los profesores, por su parte, también 
están obligados a utilizar la plataforma, el programa y los recursos, los cuales son los mismos para todos.

La incorporación de este curso obligatorio ha impactado ya en 2578 estudiantes (hasta el calendario 2015A), sin embargo, como se mencionó el curso lo dan los profesores del programa Sistemas de Información, por lo que los profesores de los otros programas permanecen al margen de estos procesos, razón por la cual habría que indagar si la inclusión de estos cursos impacta en una recepción más favorable a las TIC por parte de estudiantes y profesores.

\section{RESULTADOS}

En este trabajo presentamos los resultados obtenidos de la aplicación del cuestionario a una muestra aleatoria de 211 profesores. El cuestionario estuvo dividido en dos secciones; la primera relacionada con los obstáculos atribuibles al centro, la segunda relacionada con las barreras atribuidas al docente. La escala utilizada contemplaba los números del uno al cinco, en el que se asignaron los valores: <<no se ha iniciado, incipiente, en desarrollo, establecido, consolidado >, otorgando el valor 1 al más bajo y, 5 , al más alto; cada pregunta podía tener un valor entre 211 a 1055 puntos.

Una vez aplicado el cuestionario y procesados los datos, el puntaje más alto fue para la pregunta "Desarrollo en infraestructura y habilitación tecnológica", la cual obtuvo un porcentaje de aprobación del $64.07 \%$ (676 puntos), lo cual refleja que los profesores están de acuerdo en que el centro cuenta con un buen equipamiento e infraestructura, sin embargo, esto debe leerse con cuidado, dado que la puntuación más alta fue para el ítem "En desarrollo" (41.71\%) en el cual se señala que existe equipo e infraestructura suficiente pero es necesaria la renovación del equipo y la ampliación de la infraestructura para lograr un mayor impacto en el servicio educativo. Le sigue en la escala el indicador "Establecido" (37.27\%) que señala que se cuenta con infraestructura y equipo suficiente, así como con recursos de actualidad para otorgar servicios de calidad.

De las respuestas recabadas se obtiene que una mayoría de los profesores reconoce que el centro universitario ha invertido en equipo e infraestructura suficiente, sin embargo, también es necesaria la renovación del mismo para que puedan mejorarse los servicios educativos; esto es así, pues las aulas del centro universitario cuentan con computadoras que, en muchas ocasiones corresponden a modelos antiguos, no se les ha otorgado mantenimiento o tienen problemas mecánicos para poder iniciarse.

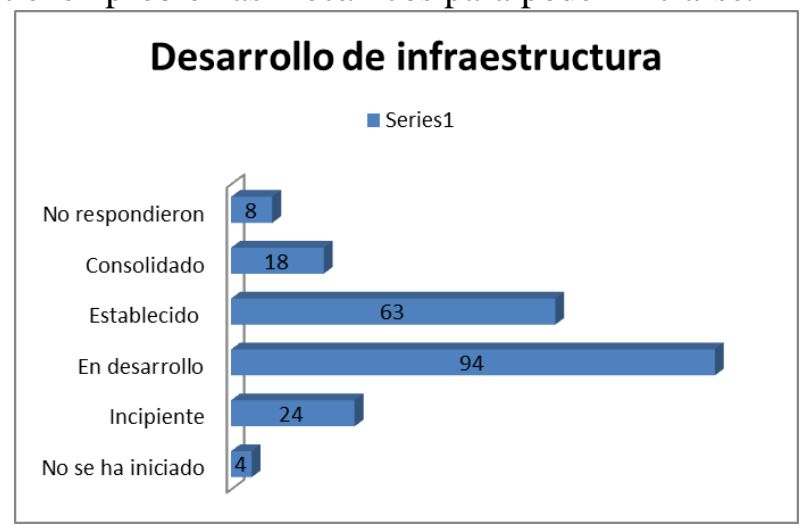

Imagen 1. Desarrollo en infraestructura y habilitación tecnológica

El segundo puntaje más alto fue para la calidad de los servicios técnicos de apoyo a la docencia, que obtuvo un porcentaje de aprobación del 59.24\% (625 puntos). Sin embargo, nuevamente debe verse con cautela dado que los profesores otorgan mayor valor al indicador 2 y 3, "Incipiente" y "En desarrollo". El "incipiente" específica que se cuenta con técnicos pero resultan insuficientes, se trabaja con apoyo de estudiantes y prestadores de servicio social. El 3, por su parte, señala que se cuenta con equipo profesional pero el número de miembros es insuficiente para otorgar servicios de calidad.

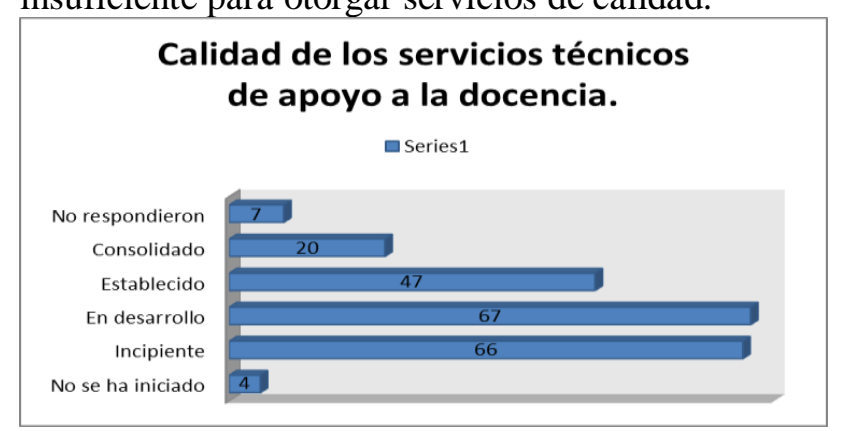

Imagen 2. Calidad de los servicios técnicos de apoyo a la docencia.

La tercera pregunta que obtuvo mayor puntuación fue la diez, relativa al uso del internet como apoyo al proceso de enseñanza aprendizaje con un porcentaje de aprobación del 59.33\% (626 puntos) y, el mayor puntaje fue para el indicador 2 "Incipiente" que señala que sólo algunos profesores utilizan el internet en el proceso de enseñanzaaprendizaje pero es a iniciativa personal y, el nivel 3 señala que se cuenta con algunos cursos y/o áreas del currículo que incorporan la TIC, pero no es una práctica generalizada.

Como focos rojos u obstáculos para integrar las TIC a las prácticas educativas $\mathrm{y}$, por ende, realizar buenas prácticas con TIC se encuentra, en primer 
lugar, la falta de "Estímulos a las prácticas pedagógicas con TIC", que obtuvo un porcentaje de aprobación del $36.20 \%$ (382 puntos). La concentración de las respuestas estuvo en los indicadores 1 y 2: "No se ha iniciado" y "En desarrollo". Con respecto al primer indicador, 101 profesores contestan que no existe ningún tipo de estímulo que incentive el uso de las TIC en las prácticas pedagógicas. En el segundo (46 puntos), los profesores dicen que se reconoce a los profesores que trabajan con TIC, se les otorga diversos apoyos como horarios flexibles, apoyo tecnológico y servicio de internet. $\mathrm{Al}$ respecto cabe comentar que, especialmente, para cursos de postgrado existen diversos apoyos cuando se utilizan las TIC pero no así en licenciatura, que abarcan la casi totalidad de los programas y, es en donde están concentrados la mayoría de los profesores. Igualmente, con sólo dos puntos de diferencia, se puede observar que la mayoría también coincide en que sólo se han otorgado estímulos pero éstos han sido de carácter material o financiero.

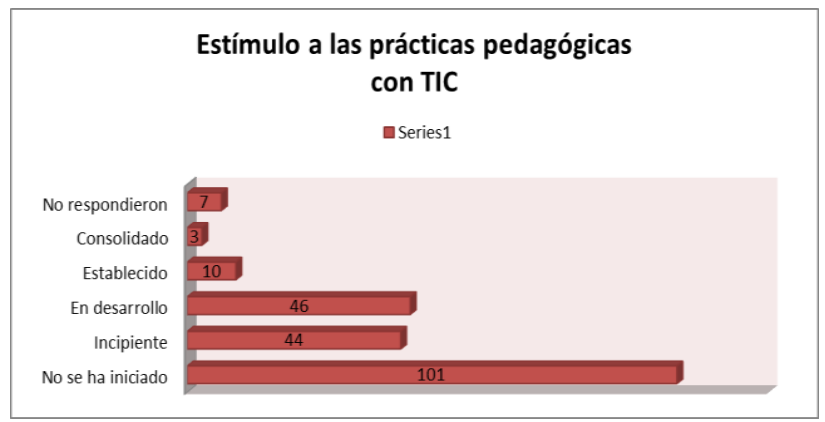

Imagen 3. Estímulo a las prácticas pedagógicas con TIC.

Otro obstáculo importante para el desarrollo de las buenas prácticas con TIC fue para la pregunta "Falta de flexibilización del diseño curricular", la cual obtuvo un porcentaje de aprobación del $45.78 \%$ (483 puntos) y, en la cual los profesores señalan que el mayor impedimento para la integración de las TIC es la falta de flexibilización de los programas curriculares, así la mayoría de las respuestas se concentran en el indicador 2 "Se trabaja con modelos presenciales, se incluyen plataformas pero no hay flexibilidad horaria".

\section{Flexibilización del diseño curricular}

ロSeries1

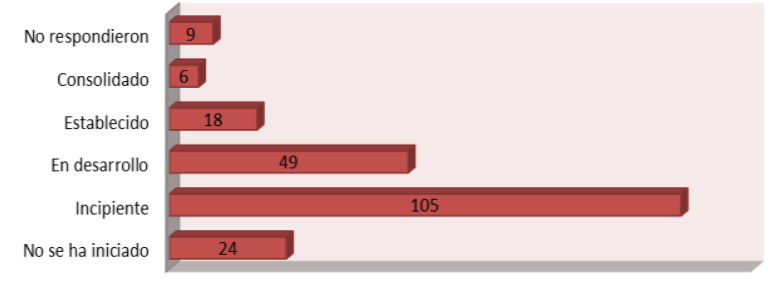

Imagen 4. Flexibilización del diseño curricular.

El otro puntaje más alto se obtuvo para el item 3: "En desarrollo" que dice que se está trabajando con modelos más flexibles que implican semipresencialidad, pero el modelo se centra más en las sesiones presenciales.

Finalmente el tercer obstáculo para la integración de las TIC a las prácticas educativas fue la "Falta de confianza en las modalidades no presenciales" que obtuvo 583 puntos y un porcentaje de aprobación del $55.26 \%$. De esta pregunta la mayor puntuación fue para el ítem 3, "En desarrollo" que dice que existe una confianza incipiente, se ha empezado a trabajar y a organizar las modalidades no presenciales en el centro. El segundo ítem con mayor peso fue para el 2 "Incipiente" que dice: hay poca confianza, existe una diferencia entre el discurso político y las prácticas reales que se observan en el centro.

\section{CONCLUSIONES}

Con la aplicación del cuestionario se contestan las preguntas de investigación planteadas:

¿Por qué no se han integrado las TIC a las prácticas educativas, considerando el alto nivel de habilitación tecnológica y de infraestructura con la que cuenta el centro universitario?

Mientras que a nivel internacional el principal obstáculo para la integración de las TIC es la falta de infraestructura y habilitación tecnológica (Bingimlas, 2009), en el CUCEA el principal obstáculo fue la falta de estímulos a las prácticas educativas. Este último se asocia con la ausencia de un horario más flexible para los profesores que implementan cursos en línea, el incentivar el uso de los cursos a través de la entrega de estímulos económicos y, la ausencia de apoyo técnico dentro del aula. En un estudio relacionado sobre la Universidad de Guadalajara López de la Madrid (2011) encontró que el $65.3 \%$ de los profesores de tiempo completo consideraban que el programa de estímulos para incentivar el uso de las TIC en la 
UdeG era deficiente. En su estudio los estímulos contemplaban los económicos, el servicio gratuito de internet y el que algunos profesores de asignatura pudieran estar excentos de registrar la entrada y la salida. Otro estudio relacionado sobre buenas prácticas con TIC desarrollado por la Coordinación de Investigación y Pregrado (CIEP, 2013) de la UdeG identificó, en un cuestionario aplicado a una muestra de los profesores, que el principal problema para la red universitaria era la falta de formación en materia de TIC, pero también se identifica como problema la falta de incentivos institucionales.

De lo anterior se desprende que el CUCEA ha apostado, principalmente, a las ayudas económicas para incentivar el uso de las TIC. De hecho el programa de estímulos académicos (apoyos salariales) otorga valor al diseño de cursos en línea, siempre y cuando sean evaluados por el Sistema de Universidad Virtual. Se ha otorgado servicio de internet gratuito para que el trabajo en línea no genere un gasto en el profesor $y$, en algunos casos se ha otorgado estímulos económicos a los profesores. Sin embargo, de acuerdo con los resultados de la investigación, los apoyos económicos no son suficientes y garantes para la integración de las TIC, por lo que la institución debe pensar en otros mecanismos para motivar a los profesores. En menor medida los incentivos han estado relacionados con la flexibilidad horaria, como lo señala López de la Madrid (ibid.), así en el CUSUR se ha aplicado esta medida a profesores de asignatura, y en el caso de CUCEA se ha otorgado flexibilidad horaria sólo a nivel posgrado y en programas cuyo contenido curricular son las propias tecnologías, tal es el caso de la Maestría en Tecnologías Educativas y la Maestría en Tecnologías de la Información.

El segundo obstáculo identificado fue la falta de flexibilización de los diseños curriculares, que de acuerdo con Area (2002) se trata de una barrera cultural, que incluye contar con diseños curriculares donde los tiempos y espacios educativos son rígidos. En este sistema se entiende que profesores y estudiantes deben coincidir en el espacio y en el tiempo para que pueda realizarse el acto educativo. Los profesores del CUCEA ven una incoherencia el trabajar con cursos en línea cuando los programas y modelos son presenciales. ¿Si se exige la presencialidad y el 100 por ciento de asistencia a las clases cuál es el propósito de usar los cursos en línea? Desde esta perspectiva se deduce que los profesores no ven los cursos en línea como una herramienta de apoyo al trabajo docente sino como un componente de trabajo extra. Para los profesores la política de incorporar los cursos en línea debe venir acompañada de una flexibilización horaria o bien de la creación de nuevos programas en modalidades semi-presenciales o, a distancia. Estas respuestas denotan que no se visualiza la integración de las TIC como una oportunidad para innovar y mejorar la práctica educativa $\mathrm{y}$, por tanto, se entiende que persisten modelos de enseñanza y prácticas tradicionales. En efecto, tal como lo señala Area las concepciones sobre la enseñanza y el aprendizaje no se modifican al incorporar las TIC e impiden, como en este caso, que se pueda visualizar el sentido de mejora que puede traer consigo el incorporar las TIC a la práctica educativa.

En tercer lugar, se agrega, de acuerdo con la revisión de la literatura latinoamericana el punto de la confianza, que efectivamente emerge como un obstáculo en la integración de las TIC a las buenas prácticas educativas, en este rubro el profesorado califica de incipiente la labor de la institución, los profesores consideran que el CUCEA transita tímidamente al trabajo con modalidades no presenciales, además de destacar la diferencia entre el discurso oficial, por lo menos lo declarado en el plan institucional $\mathrm{y}$, las prácticas reales que se observan en el centro. De acuerdo con lo anterior, esta falta de confianza puede afectar o afecta de hecho las decisiones de los policy makers, haciendo que se introduzcan estrategias que aseguren el "éxito" como lo es el asociar el uso de cursos en línea con un beneficio económico, pero se desconfíe de otras como es la flexibilidad horaria.

En relación a las barreras relativas al profesor, éstas obtuvieron muy bajo porcentaje, de esta manera, de acuerdo con los profesores los principales obstáculos son externos, deben atribuirse al centro educativo. Esto nos indica que existe una brecha entre, por una parte, las actitudes del profesorado, las cuales autocalifican de positivas y benéficas para el desarrollo de los aprendizajes de los estudiantes, pero insuficientes; los profesores, nos dicen, tienen buena disposición para trabajar con las TIC pero hacen falta programas que incentiven su participación.

Por otra parte destacan como elementos positivos dentro del CUCEA la infraestructura, sin embargo debe reconocerse que es necesaria y urgente la renovación del equipo, pues como señala Bingimlas, (2009) la falta de renovación constituye un obstáculo a la integración de la TIC. El otro punto positivo es la calidad de los servicios técnicos de apoyo a la docencia, es decir, que los profesores reconocen que hay un buen servicio, a pesar de que hace falta personal, lo cual, efectivamente convierte 
al CUCEA en un centro potencialmente avanzado en la integración de las TIC.

Finalmente, otro punto a favor del centro universitario, es el uso del internet como apoyo a la docencia, con ello se señala que existen muchos profesores que trabajan con TIC, pero lo hacen a iniciativa personal y no como una práctica generalizada, es decir, se encuentran muchos profesores motivados, con confianza en el papel que juegan las TIC, pero hace falta una buena política institucional y trabajo coordinado.

De acuerdo con lo anterior, es urgente el planteamiento de políticas orientadas a estimular las prácticas innovadoras con TIC, aprovechar la percepción positiva que tienen los profesores sobre la infraestructura y los servicios tecnológicos, de tal manera que se conviertan en un elemento a favor de su integración. Sin embargo, será necesario trabajar en dos sentidos: en la capacitación del docente sobre las TIC y, en el ámbito pedagógico para que los profesores puedan transformar su práctica educativa, pues hasta el momento los resultados marcan que a pesar de ser una política institucional los profesores no ven en el curso en línea una posibilidad para innovar su práctica educativa. La idea de innovación, en ese sentido, debe ser más amplia, no debe quedar reducida al uso e incorporación de plataformas tecnológicas, la plataforma sólo es un recurso como pueden existir otros que apoyen a transformar e innovar la práctica docente.

Algunas ideas a favor de ello pueden ser que se estimulen las prácticas educativas con TIC, a través de la organización de seminarios, congresos de buenas prácticas que permitan reconocer quiénes son esos profesores y, qué están haciendo en sus aulas. Los incentivos económicos son importantes pero también lo es el reconocimiento de pares, el cual siempre ha jugado un papel importante en la motivación de los académicos.

Igualmente será necesario ir otorgando mayor flexibilidad horaria que permita a los profesores descargar una buena parte de los créditos de su asignatura a través de su curso en línea; esto puede realizarse sin mayor conflicto, pues existe dentro de la propia universidad (Centro Universitario del Sur) experiencias en las que los profesores de un programa presencial obtienen flexibilidad horaria, siempre y cuando su curso en línea haya sido evaluado y certificado por el Sistema de Universidad Virtual, éste último sistema tiene el atributo reconocido por la Universidad de Guadalajara de acreditar y evaluar los cursos en línea que se generen en todos y cada uno de los centros universitarios.
Otro hecho positivo actual es que el centro universitario ha abierto carreras que incluyen la modalidad semi-presencial con apoyo y uso de las TIC que se habillitarán a partir de agosto de 2015 y, que constituyen un esfuerzo por realizar el tránsito hacia la flexibilización de los programas educativos. Asimismo existe el curso de Tecnologías de la Información, ahora obligatorio para todos los estudiantes, y que está tratando de desarrollar habilidades digitales para su utilización a lo largo de su trayectoria académica, habría que analizar sí efectivamente esto se realiza de esta manera.

Como líneas emergentes de investigación destaca que la investigación se centra en la política institucional de estimular el uso de cursos en línea, sin embargo no todas las TIC son cursos en línea, por tanto, deben existir profesores que integren otro tipo de tecnologías educativas a sus prácticas pedagógicas, muy posiblemente éstas tecnologías puedan estar relacionadas con el conocimiento especializado. Sobre este último punto sería conveniente identificar quiénes son esos maestros que actualmente están utilizando las TIC y cómo las incorporan a su práctica docente.

\section{DISCUSIÓN}

La aplicación del cuestionario arrojó como principal obstáculo la ausencia de incentivos. De igual manera otras investigaciones que se han realizado al interior de la UdeG demuestran que éste es una barrera para la integración de las TIC, así lo señalan las investigaciones de López de la Madrid (2011) y la CIEP (2013). Ahora bien, el problema de los incentivos está asociado a las políticas que las instituciones ponen en marcha $\mathrm{y}$, por tanto se trata de un problema administrativo. De hecho, los profesores señalan la discordancia existente entre, por una parte, lo que señalan los planes de desarrollo y las prácticas reales de integración de las TIC $\mathrm{y}$, califican las acciones que realiza la institución como incipientes. Los incentivos, hasta ahora otorgados por la institución han sido de carácter económico mientras que las demandas de los profesores apuntan a la implementación de políticas que tiene que ver con los procesos administrativos que permiten la inclusión de modelos de enseñanza aprendizaje.

Por otra parte, en una investigación con respecto a la elaboración de indicadores para la integración de las TIC Akbulut (2010) señala la política como un elemento que predice la integración de las TIC en dos grandes rubros: la infraestructura y los modelos de enseñanza aprendizaje. Con respecto a esta 
última el autor introduce las categorías de elearning, interacción y comunidades e-learning. En contraposición, Area (2002) ha manejado los modelos de enseñanza aprendizaje como un elemento cultural y, por tanto, poco permeable a las políticas educativas, ahí se encuentran elementos como prácticas educativas tradicionales, currículo rígido entre otros $\mathrm{y}$, hemos agregado, por nuestra parte, la poca confianza en los modelos no convencionales.

Se puede considerar que existe una correlación significativa entre falta de estímulos, los modelos de enseñanza aprendizaje y las políticas. Las políticas administrativas pueden estar influidas por una dimensión cultural que otorga poco valor a las modalidades educativas no tradicionales $\mathrm{y}$, por tanto genera, medidas "racionales" para promover las TIC como lo es el ofrecimiento de estímulos económicos, pero pocos cambios en la estructura administrativa que soporte la integración de las TIC, en ese sentido la relación es negativa; pero al mismo tiempo las políticas pueden verse como un factor de cambio para impulsar nuevas modalidades educativas cuando se invierte en una buena infraestructura y se apoyan acciones efectivas para impulsar el elearning, generándose con ello un cambio en la dimensión cultural.

\section{REFERENCIAS.}

Area, M. (2002). La integración escolar de las tecnologías. Entre el deseo y la realidad. Organización y gestión educativa, Revista del Forum Europeo de Administradores de la Educación, 10(6), 14-18.

Akbulut, Y. (2010). Turkish faculties of education. Regarding ICT integration indicators. Contemporary Educational Technology, 1(4), 322-334.

Barrantes, C, G., Casas G, L., y Luengo G, R. (2011). Obstáculos percibidos para la integración de las TIC por los profesores de infantil y primaria en Extremadura. Pixel-Bit. Revista de Medios y Educación, 39, 83-94. Recuperado el 20 de febrero de 2013 de: http://cdc.sav.us.es/pixelbit/images/stories/p39/07.pdf

Bingimlas, K, A. (2009) Barriers to the successful integration of ICT in teaching ad learning environments: A review of the literature. Euroasia Journal of Mathematics, Science \& Technology Education, 5(3), 235-245. Recuperado el 08 de mayo de 2013 de: http://www.ejmste.com/v5n3/eurasia_v5n3_bingimlas.p df

Cabero, J. (2001). Tecnología educativa. Diseño y utilización de medios en la enseñanza. Barcelona: Paidós.

CIEP (2013). Encuesta de prácticas docenes innovadoras. Guadalajara: México, Universidad de Guadalajara, Informe.

CUCEA (2002). Plan de Desarrollo Institucional 2002-2010. Jalisco, México: Universidad de Guadalajara. Recuperado de: http://www.copladi.udg.mx/planeacion/pdi
CUCEA (2013) Unidad de Diseño Educativo UDE Antecedentes. Recurso en línea: http://www.cucea.udg.mx/?q=cta/ude

CUCEA (2014a). Actualización del Plan de Desarrollo Institucional del Centro Universitario de Ciencias Económico Administrativas. Visión 2030. Jalisco, México: Universidad de Guadalajara. Recuperado de: http://www.cucea.udg.mx/sites/default/files/documentos /adjuntos_pagina/pdi-2014-2030.pdf

CUCEA (2014b). Numeralia CUCEA. Información $e$ indicadores básicos. Zapopan, México. Universidad de Guadalajara. Recuperado de: http://www.cucea.udg.mx/sites/default/files/documentos /numeralia/numeralia_cucea_marzo_2014.pdf

CUCEA (2015). Numeralia CUCEA. Información e indicadores básicos. Zapopan, México. Universidad de Guadalajara. Recuperado de: http://www.cucea.udg.mx/sites/default/files/documentos /numeralia/numeralia_cucea_1er_cuatrimestre_2015.pdf

CUSUR (2014). Manual para el uso y manejo de los ambientes virtuales de aprendizaje del Centro Universitario del Sur. Ciudad Guzmán, México. Universidad de Guadalajara. Recuperado de: http://www.cusur.udg.mx/es/manual-deprocedimientos-evas

Gobierno de la República (2007). Plan Nacional de Desarrollo 2007-2012. México: Poder Ejecutivo Federal. Recuperado de: http://www.oic.sep.gob.mx/portal3/doc/PMG/pnd_2007 -2012.pdf

Gobierno de la República (2013). Plan Nacional de Desarrollo 2013-2018. México: Poder Ejecutivo Federal. Recuperado de: http://www.snieg.mx/contenidos/espanol/normatividad/ MarcoJuridico/PND_2013-2018.pdf

Jones, A., (2004). A Review of the research literature on barriers to the uptake of ICT by teachers. British Educational Communications and Technology Agency, (BECTA). Recuperado el 21 de febrero de 2013 de: http://dera.ioe.ac.uk/1603/1/becta_2004_barrierstouptak e litrev.pdf

López de la Madrid, M. C. (2011). El cambio en las Universidades a partir del uso de las tecnologías de la información y la comunicación. Análisis de procesos desde un estudio de caso. Tesis doctoral.

Olavarria-Gambi, M., Carpio, C. (2006). Educación a distancia y universidades. La experiencia de la GDLN. Revista de Educación a Distancia, 16. Recuperado el 09 de enero de 2015 de: http://www.um.es/ead/red/16

Padilla, S., Moreno, C., Bravo, I. (2015). Factores generating barriers to the integration process of ICTs in Higher Education Institutions. Case study in a education center of the University of Guadalajara. International Journal of Instructional Tecnology \& Distance Learning, 12(3), 39-52,

Pelgrum, W, J. (2001). Obstacles to the integration of ICT in education: results from a worldwide educational assessment. Coumputers \& Education, 37, 163-178. Recuperado el 24 de marzo de 2013 de: http://users.ntua.gr/vvesk/ictedu/article5_pelgrum.pdf

Ramírez, E. Cañedo, I., Clemente, M. (2011). Las actitudes y creencias de los profesores de secundaria sobre el uso del Internet en sus clases. Comunicar, XIX(38), 47-155. Recuperado el 11 de febrero de 2013 de: http://www.revistacomunicar.com/pdf/preprint/38/16PRE-12643.pdf 
Secretaría de Educación Pública (2007). Plan sectorial de Educación 2007-2012. México: Secretaría de Educación Pública. Recuperado de: http://www3.utvm.edu.mx/wpcontent/uploads/2015/06/Programa-Sectorial-20072012.pdf

Secretaría de Educación Pública (2013). Plan sectorial de educación 2013-2018. México: Secretaría de Educación Pública. Recuperado de: http://www.sep.gob.mx/work/models/sep1/Resource/44 79/4/images/PROGRAMA_SECTORIAL_DE_EDUCA CION_2013_2018_WEB.pdf
Valzacchi, J. (2010). Educación virtual en Argentina. De dónde venimos y hacia dónde deberíamos ir (corrigiendo algunos errores). En P. Lupión y C. Rama (Coords.). La educación superior a distancia en América Latina y el Caribe, (pp.17-24). Editora UNISUL.

Vianney, J., Lupión, T. (2010). La educación a distancia en el Brasil. En C. Rama y J. Pardo (Coords.), La educación superior a distancia: Miradas diversas desde Iberoamérica, (pp.22-54) Madrid, Instituto Tecnológico Virtual de Educación. 